\title{
Interfacial and Emulsifying Properties of Diacylglycerol
}

\author{
Atsuko SHIMADA and Kyoko OHASHI \\ Graduate School of Human Life Science, Showa Womens University, Setagaya-ku, Tokyo 154-8533, Japan
}

Received May 27, 2002; Accepted January 29, 2003

\begin{abstract}
The interfacial properties and emulsifying properties of diacylglycerol (DAG) were examined for comparison with those of triacylglycerol (TAG). The fatty acid composition and other properties of DAG were adjusted to approximately the same levels as those of TAG. The interfacial tension of DAG was about half that of TAG. When DAG was mixed with TAG, the interfacial tension of the mixture decreased in proportion to the increasing concentration of DAG and showed no breaking point. Mixtures of DAG and water were homogenized with varying oil-water proportions, with and without salt, and with and without an emulsifier. DAG was more easily emulsified than TAG and tended to become a water/oil (w/o) emulsion. The addition of salt markedly increased the stability of the DAG emulsion. DAG mixtures containing $0.25 \%$ of an emulsifier having an HLB value of between 4.5 and 13 formed w/o emulsions, while o/w emulsions were formed with TAG under the same conditions.
\end{abstract}

Keywords : interfacial property, emulsifying property, diacylglycerol, salt, emulsifier

Diacylglycerol (DAG) consists of one glycerol and two fatty acids, attached to each other by an esterific bond. It has the unique property that the components after digestion are hard to convert into neutral fat in the body, so that less fat is accumulated under the skin than with triacylglycerol (TAG) (Nagao \& Naito, 1999; Nagao et al., 2000; Yasukawa \& Yasunaga, 2001). DAG was approved in 1998 as a food for specified health uses by the Japanese Ministry of Health, Welfare and Labor and is believed to help prevent the lifestyle related diseases that are thought to be derived from obesity. Fats and oils are commonly used for dressings, deep-frying and pan-frying. However, little is known about the cooking properties of DAG, although Ogawa et al. (2001) reported that the quality of cakes prepared with DAG-rich oil was almost the same as those prepared with TAG-rich oil. We earlier reported that the stability of DAG with regard to autoxidation was the same as that of TAG (Nakatsugawa et al., 2000). Simulated-mayonnaise o/w emulsions prepared with DAG had higher viscosity and smaller oil droplets than those with TAG (Ohashi \& Shimada, 2001), implying that less oil was needed to obtain the desirable viscosity for mayonnaise. Moreover, even if the size of the oil droplets in emulsions prepared with DAG was adjusted to almost the same as that with TAG, the viscosity of the emulsions prepared with DAG was higher. It is reported that DAG is useful as a texture-improving agent in starchy food (Sugiura et al., 1990) and in w/o/w emulsions (Suzuki et al., 1991), and as a coagulating agent in tofu preparation (Suzuki et al., 1993). These features suggest that DAG has emulsifying properties different from those of TAG.

The aim of this study was to clarify the characteristics of the interfacial properties and emulsifying properties of DAG for comparison with those of TAG in simple oil and water systems.

\section{Materials and Methods}

Materials DAG and TAG oil samples were prepared by

E-mail: shimada@swu.ac.jp
Kao Corporation according to the methods outlined in a previous paper (Nakatsugawa et al., 2000). The glyceride composition and other properties of each oil are shown in Table 1. More details about the properties have been presented in a previous paper by Ohashi and Shimada (2001). The fatty acid composition and the tocopherol contents of the DAG sample were adjusted to approximately the same levels as those of the TAG sample. These samples were used without further purification to evaluate the emulsifying properties and the water-holding capacity. Portions of the DAG and TAG oil samples were purified by silica gel column chromatography using a series of hexane and ethyl acetate eluting solutions. After trimethylsilylation, a gas liquid chromatograph with an FID detector was used to check the purity level of the samples and it was found to be over $99.9 \%$. These purified samples and glycerol monooleate (MAG; Sigma Chemicals, MO) were used for measuring the interfacial tension. Polyglycerol oleic acid esters with hydrophile-lipophile-balance (HLB) values ranging from 3 to 14.5 (Taiyo Kagaku, Mie; Table 4) were used as emulsifiers, and Sudan red III (super grade; Kanto Kagaku, Tokyo) was used as a coloring agent in the oil samples. The $\mathrm{NaCl}, \mathrm{KCl}, \mathrm{MgCl}_{2}$ and $\mathrm{CaCl}_{2}$ reagents were of super grade, and deionized water was used throughout the experiments.

Measurement of the interfacial tension The interfacial tension of the purified DAG and TAG samples was measured with a TVT1 drop-volume tensiometer (Lauda, Germany) at $25^{\circ} \mathrm{C}$.

Preparation of emulsions and evaluation of their emulsifying properties The unpurified DAG and TAG oil samples were stained with $0.05 \%$ (w/v) Sudan red III before emulsification. A $20-\mathrm{ml}$ volume of oil and water was mixed at 10,000 rpm for 5 min with an Excel ED-2 homogenizer (Horiba Seisakusho, Kyoto), at $20^{\circ} \mathrm{C}$ (Table 2), then three variables were introduced into the composition of each emulsified sample. First, the oil-volume fraction $(\phi)$ was varied from 0.1 to 0.9 in increments of 0.1 . Second, $0,0.05,0.1,0.2$ and $0.5 \mathrm{M}$ aqueous solutions of $\mathrm{NaCl}, \mathrm{KCl}$, $\mathrm{MgCl}_{2}$ or $\mathrm{CaCl}_{2}$ were used as the aqueous phase instead of water 
with an oil-volume fraction of 0.5 to prepare 20 kinds of emulsion. Third, emulsifiers having HLB values ranging from 3 to 14.5 (Table 4) were included with the components for emulsification at an oil-volume fraction of 0.5 .

Emulsifiers with an HLB value ranging from 3 to 10 were dissolved in the DAG and TAG oils at concentrations of $0.25,0.5$, 1.0, 2.0, 3.0, 4.0 and 5.0\% (w/v), while emulsifiers with HLB values of 13 and 14.5 were dissolved in water at the same concentrations, resulting in 56 kinds of emulsions prepared with oil, water and the emulsifier. All emulsifiers were dissolved in oil or water at $60^{\circ} \mathrm{C}$ and emulsification was conducted at $20^{\circ} \mathrm{C}$. The type of each prepared emulsion was evaluated just after preparation using a diluting method (Kunieda \& Aramaki, 2001). A 10$\mathrm{ml}$ aliquot of each emulsion was transferred to a graduated test tube with a cap and stored for up to 7 days at $20^{\circ} \mathrm{C}$, and changes with time in the volume of the emulsified layer, the aqueousphase layer and the oil-phase layer were measured. The volume of the emulsified layer remaining represented the stability of the emulsion.

Measurement of the water-holding capacity Each oil sample $(10 \mathrm{ml})$ was mixed at $820 \mathrm{rpm}$ with an MK-13 hand mixer (Matsushita Electric, Kobe) at $20^{\circ} \mathrm{C}$, while water was added at the rate of $0.1 \mathrm{ml} / \mathrm{s}$. Mixing was continued until no more water could be dispersed in the oil-water mixture when it formed a gel.

Table 1. General properties of the TAG and DAG oil samples.

\begin{tabular}{|c|c|c|c|}
\hline \multirow{2}{*}{ Property } & & \multicolumn{2}{|c|}{ Sample } \\
\hline & & TAG & DAG \\
\hline \multicolumn{4}{|l|}{ Fatty acid composition (\%) } \\
\hline & C $16: 0$ & 5.7 & 3.1 \\
\hline & C 18:0 & 2.2 & 1.3 \\
\hline & C $18: 1$ & 36.2 & 37.8 \\
\hline & C $18: 2$ & 46.7 & 48.6 \\
\hline & C $18: 3$ & 8.2 & 8.5 \\
\hline & C 20:1 & 0.9 & 0.7 \\
\hline & Total & 100.0 & 100.0 \\
\hline Tocopherol content (ppm) & & 1058 & 1029 \\
\hline \multicolumn{4}{|l|}{ Acylglycerol composition (\%) } \\
\hline & MAG & 0.2 & 1.0 \\
\hline & DAG & 1.4 & 87.1 \\
\hline & TAG & 98.4 & 11.9 \\
\hline & Total & 100.0 & 100.0 \\
\hline Specific gravity $\left(20^{\circ} \mathrm{C}\right)$ & & 0.914 & 0.926 \\
\hline Surface tension $\left(25^{\circ} \mathrm{C}\right)$ & $(\mathrm{mN} / \mathrm{m})$ & 35.0 & 34.5 \\
\hline Viscosity $\left(20^{\circ} \mathrm{C}\right)$ & $(\mathrm{mPa} \cdot \mathrm{s})$ & 63.8 & 78.6 \\
\hline
\end{tabular}

The volume of water that had been dispersed in the oil divided by the volume of oil $(10 \mathrm{ml})$ is defined as the water-holding capacity of the oil.

\section{Results and Discussion}

Interfacial activity of DAG DAG is considered likely to have interfacial activity since it has a hydrophobic moiety derived from fatty acids and a hydrophilic moiety derived from the $\mathrm{OH}$ group in the molecule. The fact that DAG has a HLB value of about 2.8 when it is applied to Griffins Equation supports this estimation. The interfacial tension of the DAG sample was about one half that of the TAG sample (Fig. 1), although the surface tension of the two (Table 1) was almost the same. Fujii et al. (1985) reported that the interfacial tension of diolein was lower than that of triolein. The change in interfacial tension of TAG after the addition of DAG was examined using the purified DAG and TAG oils (Fig. 1). The interfacial tension of the TAG oil decreased in proportion to the increase in DAG concentration, but it showed no breaking point. On the other hand, the addition of only $0.1-1.0 \%$ of glycerol monooleate (MAG) markedly reduced the interfacial tension of the TAG oil and showed a breaking point at about $2 \%$. The curve for the TAG-MAG mixtures is similar to the "Gibbs adsorption plot", though this is applied to

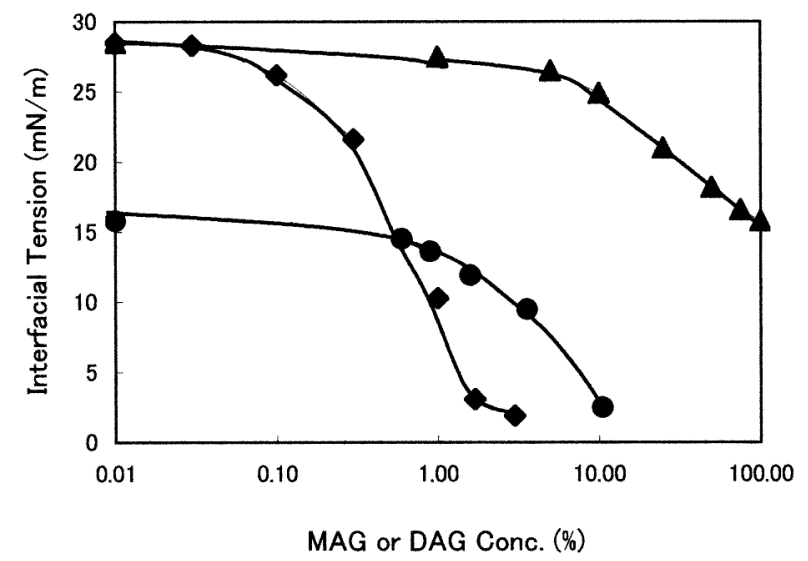

Fig. 1. Effects of the concentration of MAG or DAG on interfacial tension of TAG and of the concentration of MAG on interfacial tension of DAG at $25^{\circ} \mathrm{C}$. $\boldsymbol{\Delta}$, DAG concentration in TAG; $\mathbf{\square}$, MAG concentration in TAG; MAG concentration in DAG.

Table 2. Composition of each emulsion.

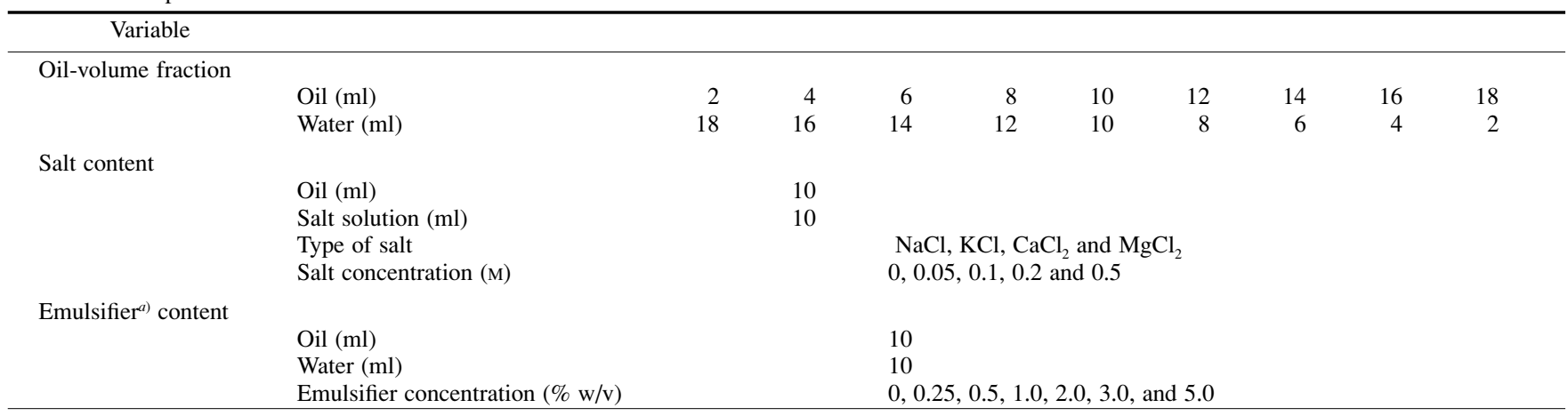

a) Type of emulusifiers are shown in Table 4. 
the surface tension of air-water interface, and the breaking point is referred to as the critical micelle concentration. It is suggested that MAG exists in a soluble state in TAG and a part of it was adsorbed at the interface between TAG and water. This adsorbed part is believed to increase with the increase in concentration of MAG and to reach maximum adsorption at about $2 \%$.

The adsorption to the oil-water interface of an emulsifier dissolved in a non-polar solvent is generally influenced by the solvation of the hydrophobic moiety in the emulsifier, and by the strength of the hydrogen bonds of the hydrophilic moiety in the emulsifier. The affinity of DAG and MAG for TAG, which is a non-polar solvent, is thought to be influenced by the chain length of the fatty acids and by the number of fatty acids present. Since DAG and MAG both consist mainly of carbon-18 fatty acids, their affinity for TAG, resulting from the chain length of the fatty acids, is thought to be almost the same. However, the affinity of DAG for TAG derived from the number of fatty acids is stronger than that of MAG, because DAG has two fatty acids in the molecule while MAG has only one. Moreover, the affinity of DAG for water, resulting from the number of $\mathrm{OH}$ groups, is weaker than that of MAG. It seems that DAG dissolved in TAG because of a strong affinity for TAG and a weak affinity for water.

When MAG was mixed with DAG, the interfacial tension of the mixture decreased with increasing concentration of MAG. However, the lowering effect of MAG on the interfacial tension of DAG was less than the effect on TAG. About ten times greater concentration of MAG was needed to reduce the interfacial tension of DAG than that needed to reduce the interfacial tension of TAG. (Since the DAG-MAG mixture formed crystals above a $10 \%$ concentration of MAG, we could not measure the interfacial tension above $10 \%$ ). It is thought that MAG is more soluble in DAG than in TAG, because the polarity of MAG is closer to that of DAG than that of TAG.

It is clear that DAG had different interfacial properties from those of TAG, and the results obtained appeared to indicate that the emulsifying properties of DAG were different from those of TAG and could influence the rheological properties during food processing and the quality of various food products.

Characteristics of the emulsifying properties of DAG Figure 2 shows the type of emulsions formed with TAG and DAG, and the stability of the emulsions as a function of the oil-volume fraction $(\phi)$. The TAG mixtures with $\phi$ of $0.1,0.2$ and 0.3 became $\mathrm{o} / \mathrm{w}$ emulsions just after being homogenized, while the mixtures with $\phi$ of 0.4 and above became w/o emulsions. All the mixtures began to separate as soon as mixing was stopped, and separated almost completely into an oil phase and aqueous phase over the next $7 \mathrm{~h}$. In contrast, all the DAG mixtures formed the w/o type of emulsion, those with $\phi$ of 0.3 and above becoming homogeneous w/o emulsions. The mixture with $\phi$ of 0.1 , which contained $90 \%$ water, formed an emulsion layer of about $20 \%$ of the mixture and an aqueous layer of about $80 \%$. This remaining aqueous layer, which had not been dispersed into the emulsion, was also present in the mixture with $\phi$ of 0.2 .

The stability of the emulsions prepared with DAG was much higher than that of the emulsions with TAG. The volume of the emulsion layer in the mixture having an oil-volume fraction of 0.3 did not decrease at all after standing $24 \mathrm{~h}$, indicating that the mixture had retained $100 \%$ of the emulsion layer. The amount of water dispersed into the emulsion was $70 \%$ of the total volume of the emulsion, which is close to a close-packing ratio. This emulsion was very viscous, this probably being the reason for its high stability. DAG gradually separated over time in the emulsions with $\phi$ of 0.4 and higher, the amount of separated oil increasing as the oil-volume fraction was increased. However, no water separated from any of the mixtures until they had been standing for over $24 \mathrm{~h}$ as shown in Fig. 2-B. After standing for 24 $\mathrm{h}$, an aqueous layer appeared in the mixtures with $\phi$ of 0.3 and above, and the volume of this layer increased gradually while standing. After standing for 4 days, only the mixtures with $\phi$ of 0.5 and 0.6 retained the emulsion layer, as shown in Fig. 2-B. The emulsion layer in the mixture with $\phi$ of 0.6 disappeared after standing for 6 days. After 7 days, all the mixtures had completely separated into an aqueous layer and an oil layer, as shown in Fig. 2-B.

The volume of the aqueous layer in the mixtures with $\phi$ of 0.1 , which had not been dispersed into the emulsions by mixing, did not increase with $7 \mathrm{~h}$ of standing, while that with $\phi$ of 0.2 began to increase after standing $2 \mathrm{~h}$ (figure not shown).

An emulsion usually can only be formed in the presence of an emulsifier, which adsorbs at the oil-water interface and prevents coalescence of the dispersed droplets. It was found, however, that DAG was able to form a stable emulsion without the aid of an emulsifier and that DAG tended to form a w/o emulsion with various oil-volume fractions.

These findings prompted an experiment to investigate how much water could be dispersed into the DAG oil. To evaluate the water-holding capacity of DAG in comparison with TAG, $10 \mathrm{ml}$ of each oil was mixed while water was slowly added. The emulsion prepared with TAG separated suddenly after $9.7 \mathrm{ml}$ of water had been added, while that prepared with DAG did not separate, even when about 6 times the amount of water had been added. When more water was added, the water remained outside the emulsion as droplets, although mixing was continued. The waterholding capacity of DAG was recorded as 5.83 (Table 3), at which point the emulsion contained $85 \%$ water and had formed a gel.

The dispersed particles in an emulsion generally exist in a globular form when the amount of dispersed particles is below $74 \%$ of the total amount of the emulsion. When the amount of dispersed particles exceeds $74 \%$, which is the close-packing ratio, phase inversion occurs in the emulsion, or otherwise the dispersed particles become polygon-shaped and the emulsion forms a gel. It was found that DAG produced a gel emulsion containing dispersed water above the close-packing ratio.

When Griffins Equation is applied to DAG, the resulting HLB value is approximately 2.8 , as mentioned earlier. These results suggest that DAG behaves not only as an oil in emulsification, but also as an emulsifier with a low HLB value. With regard to the effect of the molecular structure of an oil on its emulsifying properties, it has been reported that the longer the fatty acid chain length and the greater the number of double bonds, the higher the stability of the resulting emulsion (Kimura et al., 1994); however, triglycerol and an emulsifier were used in that study. There has been no previous report on an emulsion prepared without the aid of an emulsifier, as we report here.

Effect of added salt on the emulsifying properties of DAG Salt, which is an essential seasoning in food processing, is known to often affect the stability of an emulsion, because the 
salt ions in the aqueous phase change the ionic state at the surface of the dispersed globules in the emulsion. The most common salt used for seasoning is $\mathrm{NaCl}$, although other salts like
$\mathrm{KCl}, \mathrm{CaCl}_{2}$ and $\mathrm{MgCl}_{2}$ are often added to $\mathrm{NaCl}$ in order to give a more complex taste and/or to reduce the intake of $\mathrm{NaCl}$. The effect of adding these four salts on the stability of the emulsions

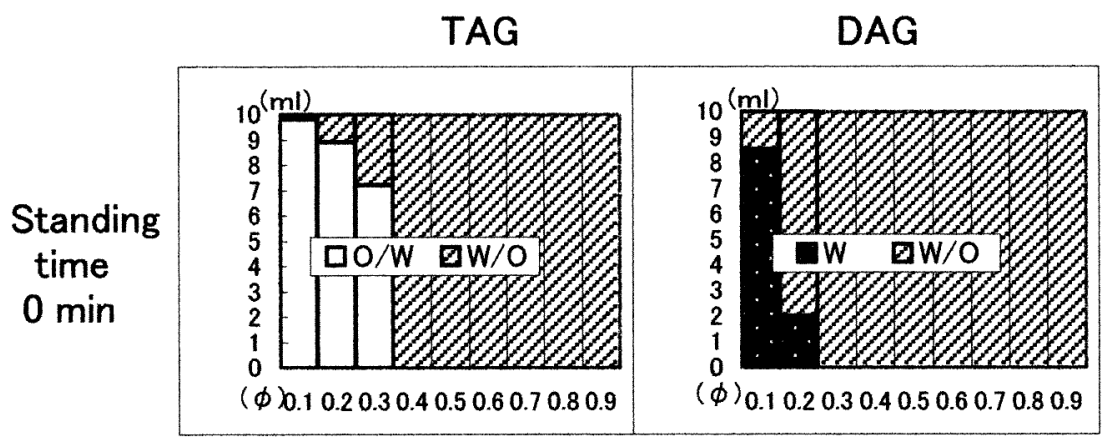

(A)

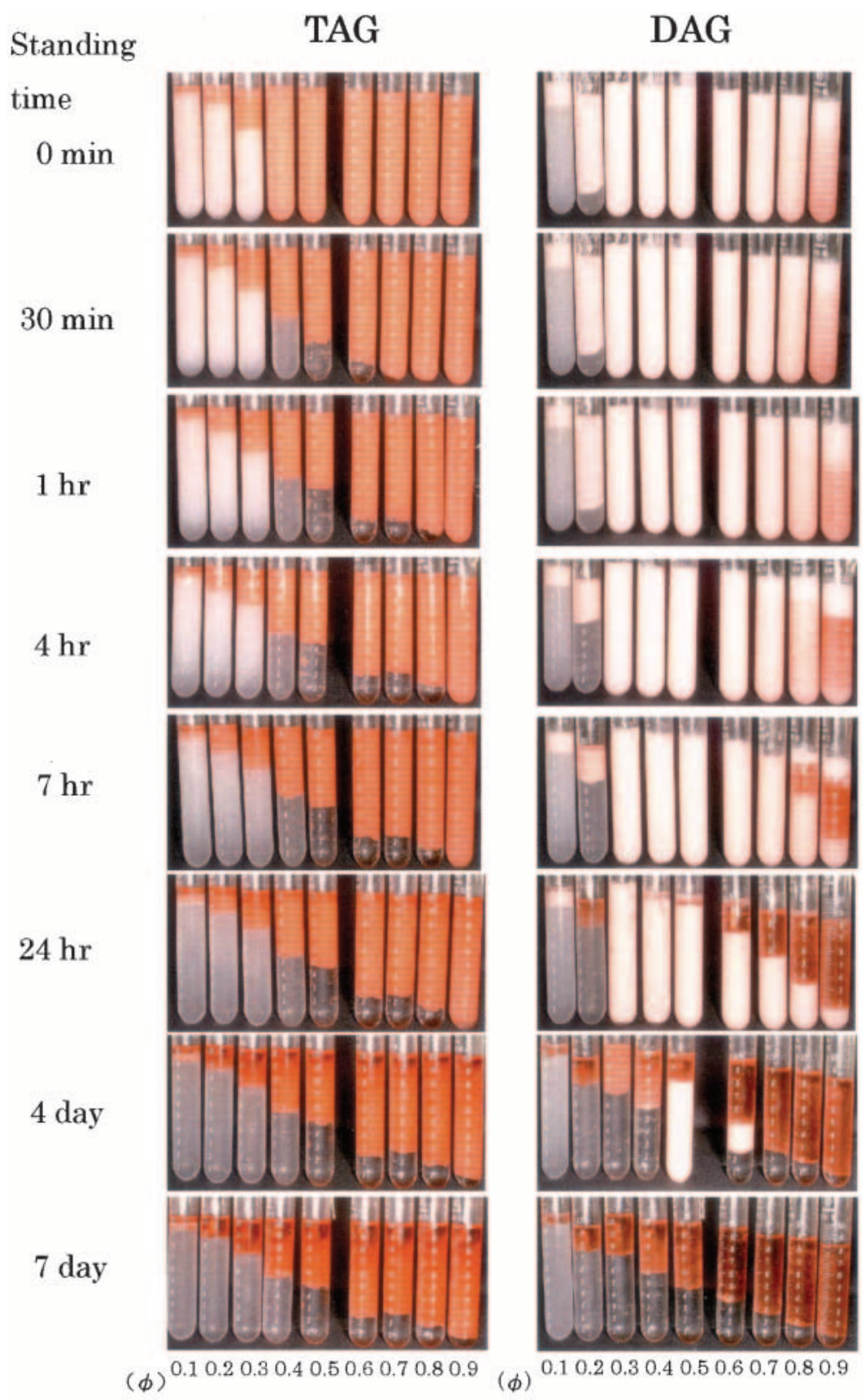

(B)

Fig. 2. Effects of the oil-volume fraction on the type of emulsion prepared with TAG and with DAG (A), and on stability of the resulting emulsion during standing at $20^{\circ} \mathrm{C}(\mathrm{B})$. 


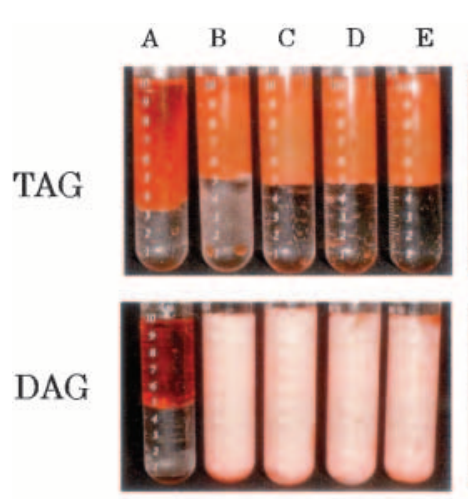

$\mathrm{NaCl}$
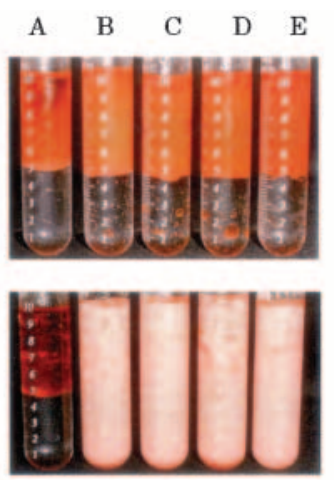

$\mathrm{KCl}$
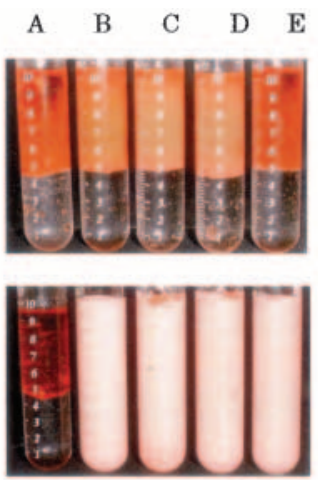

$\mathrm{MgCl}_{2}$
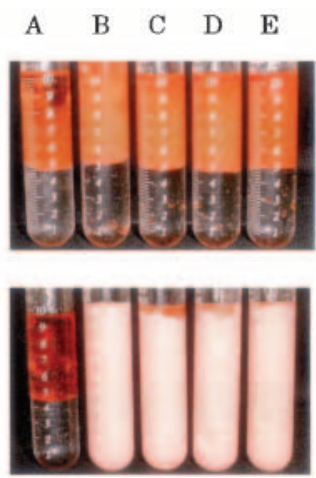

$\mathrm{CaCl}_{2}$

Fig. 3. Effects of various concentrations of salt solutions on the stability of the TAG and DAG emulsions after standing for 7 Days at $20^{\circ} \mathrm{C}$. A, $0 \mathrm{M}$; B, $0.05 \mathrm{M} ; \mathrm{C}, 0.1 \mathrm{M} ; \mathrm{D}, 0.2 \mathrm{M} ; \mathrm{E}, 0.5 \mathrm{M}$.

Table 3. Water-holding capacity of TAG and DAG.

\begin{tabular}{cc}
\hline TAG & DAG \\
\hline 0.97 & 5.83 \\
\hline
\end{tabular}

Water-holding capacity $=$ volume of water dispersed in the oil $(\mathrm{ml}) /$ volume of oil (ml)

was therefore examined. All mixtures prepared with DAG and salt solutions formed w/o type emulsion. Each of these salts substantially increased the stability of the emulsions prepared from DAG (Fig. 3). The emulsions containing each of the salts did not separate at all after standing for 7 days, although the emulsion without any salt had completely separated into oil and water after the same period. The effects of the type of salt and its concentration on the stability of the emulsion were not clear. Since the $\mathrm{OH}$ groups in DAG molecules are thought to associate with the water droplets dispersed in an emulsion and thus to contribute to the stability of the emulsion, it is probable that interaction between the $\mathrm{OH}$ groups and salt ions imparted stability to the emulsion structure. The effect of added salts on the stability of w/o emulsions prepared with TAG and a surfactant having a low HLB value has been reported by Suzuki and Tsutsumi (1989). They explained that the added salts might change the structure of liquid crystals forming the films at the oil/water interface. Further experiments are in progress to clarify the effect of added salts on the stability of emulsions prepared with DAG.

Effect of an emulsifier on the type of emulsion produced A w/o type of emulsion is usually produced when an emulsifier with an HLB value in the range of about 3.5 to 6 is used, while an o/w type of emulsion results from an emulsifier with an HLB value of 8 to 18 . The relationship between the HLB value of the emulsifier and the type of emulsion formed was therefore examined with polyglycerol fatty acid esters having an HLB value of 3 to 14.5. TAG produced w/o type emulsions when emulsifiers with HLB values of 3 and 4 were used, while o/w emulsions resulted from emulsifiers with HLB values of 4.5 and above as shown in Table 4 . The type of emulsion formed was not affected by the concentration of the emulsifier. When the emulsifiers were used at concentrations of $1.0 \%$ and above, the type of emulsion prepared with DAG was the same as that with TAG; that is, the emulsions prepared with an emulsifier of less than 4.5 HLB were of the w/o type. However, when the emulsifier was used at a concentration of $0.25 \%$ with DAG, most of the emulsions were of the w/o type, as shown in Table 4, the only exception being the emulsion prepared with the addition of an emulsifier with an HLB value of 14.5. The emulsions prepared with the 13.0 and $14.5 \mathrm{HLB}$ emulsifiers at a $0.5 \%$ concentration were of the $\mathrm{o} / \mathrm{w}$ type, while the other emulsions were of the w/o type.

It is clear that forming an o/w emulsion with DAG is rather difficult, and that a high concentration of an emulsifier would be needed. These results seem quite reasonable when we take into

Table 4. Difference between the type of emulsion prepared with TAG and DAG using emulsifiers with various HLB values at various concentrations.

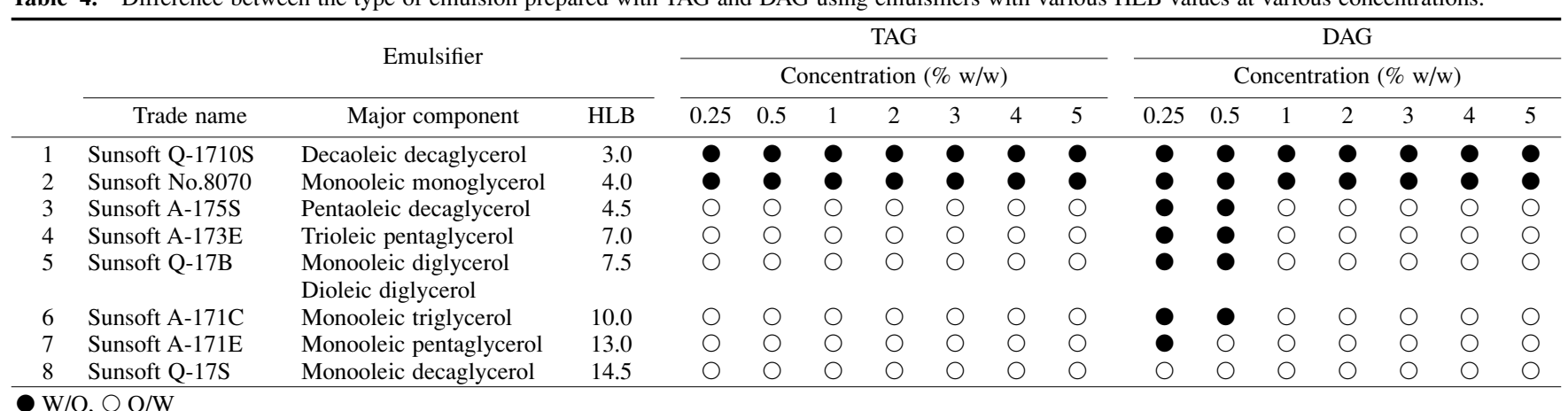


account the low HLB value of DAG obtained in Griffins Equation.

This new knowledge will provide useful information for studies in food science and for food processing.

Acknowledgments The authors appreciate the help of Mr. Yoshinobu Nakajima, senior researcher in the Health Care Products Research Laboratory of Kao Corporation, in leading this study, and the cooperation of Miss Michiko Takahashi and Miss Satomi Matsumoto. They also thank Kao Corporation for providing the DAG and TAG samples, and Taiyo Kagaku for supplying the emulsifiers.

\section{References}

Fujii, T., Shiraishi, Y. and Kawase, T. (1985). Effect of glycerides on the interfacial tension at an oil/water interface. Yukagaku, 34, 446450 (in Japanese).

Kimura, M., Shizuki, M., Miyoshi, K., Sakai, T., Hidaka, H., Takamura, H. and Matoba, T. (1994). Relationship between the molecular structures and emulsification properties of edible oils. Biosci. Biotechnol. Biochem. 58, 1258-1261.

Kunieda, H. and Aramaki, K. (2001). Emulsion and HLB. Oleo Sci., 1, 179-186 (in Japanese).

Nagao, T. and Naito, S. (1999). Nutritional effect of diacylglycerol as cooking oil. Nihon Shokuseikatsu Gakkaishi, 10, 19-26 (in Japanese).

Nagao, T., Watanabe, H., Goto, N., Onizawa, K., Taguchi, H., Matsuo,
N., Yasukawa, T., Tsushima, R., Shimazaki, H. and Itakura, H. (2000). Dietary diacylglycerol suppresses accumulation of body fat compared to triacylglycerol in men in a double-blind controlled trial. J. Nutr., 130, 792-797.

Nakatsugawa, K., Ohashi, K. and Shimada, A. (2000). Comparison of oxidative stability of diacylglycerol and triacylglycerol. Nippon Shokuhinn Kagaku Kogaku Kaishi, 48, 429-436 (in Japanese).

Ogawa, H., Okushima, S. and Kodama, H. (2001). The cooking characteristics of diacylglycerol rich cooking oil when preparing confectionary. Nihon Shokuseikatsu Gakkaishi, 12, 36-42 (in Japanese).

Ohashi, K. and Shimada, A. (2001). Emulsifying properties of diacylglycerol in a concentrated oil-in-water emulsion system. Nihon Chorikagaku Kaishi, 35, 132-138 (in Japanese).

Sugiura, S., Suzuki, K., Kasuga, Y., Inaoka, S., Yoshida, H., Maeda, H. and Ito, H. (1990). Quality improving agents in starchy food. Japan Kokai Tokkyo Koho, 02-124052. May 11 (in Japanese).

Suzuki, K., Uehara, K. and Ohmura, H. (1991). Oil composite in a state of o/w/o double emulsion. Japan Kokai Tokkyo Koho, 03236743. Oct. 22 (in Japanese).

Suzuki, K., Yoshida, H. and Kanai, K. (1993). Coagulating agents in tofu preparation. Japan Kokai Tokkyo Koho, 05-304923. Nov. 19 (in Japanese).

Suzuki, Y. and Tsutsumi, H. (1989). Effect of inorganic salts on the stability of w/o emulsions stabilized by 1-o-alkylglycerol. Nippon Kagaku Kaishi, 10. 1693-1699 (in Japanese).

Yasukawa, T. and Yasunaga, K., (2001). Nutritional function of dietary diacylglycerol. J. Oleo Sci., 50, 427-432. 